REVISTA ECONOMÍA

Vol. 69, N. ${ }^{\circ}$ Io9 (mayo), 79-98

\title{
EXPERIENCIAS DE VINCULACIÓN DE UNIVERSIDADES PÚBLICAS CON ORGANIZACIONES Y MOVIMIENTOS DE ECONOMÍA SOCIAL Y SOLIDARIA DE ARGENTINA Y PERÚ
}

\author{
LUIS MONTOYA \\ Universidad Nacional Mayor de San Marcos
}

Recepción manuscrito: 6 de enero de 2017

Aceptación versión final: 15 de abril de 2017

\begin{abstract}
RESUMEN El trabajo analiza las experiencias del Programa de extensión universitaria Construyendo redes emprendedoras en Economía social de la Universidad Nacional de Quilmes, del Gran Buenos Aires, Argentina; y el Seminario de Economía social, solidaria y popular de la Universidad Nacional Mayor de San Marcos, de Lima, Perú. Experiencias de investigación acción, extensión universitaria y formación, gestionadas desde universidades públicas interesadas en desenvolver vinculaciones con organizaciones y movimientos de economía social y solidaria de Argentina y Perú.
\end{abstract}

PALABRAS CLAVE Economía social y solidaria, universidad pública, Perú, Argentina.

ABSTRACT This article analyzes the experiences of the University extension program Building entrepreneurial networks in Social economy at the National University of Quilmes, Greater Buenos Aires, Argentina, and the Seminar of Social, Solidarity and Popular Economy at the National University of San Marcos, Lima, Peru. Experiences of research action, university extension and training, managed from public universities interested in developing links with organizations and social and solidarity economy movements of Argentina and Peru.

KEYWORDS Social and solidarity economy, public university, Peru, Argentina.

JEL CODES R13, L31.

\section{INTRODUCCIÓN}

La cinematografía muchas veces puede proyectar no solo imágenes que expresan las sensibilidades predominantes, propias de una determinada realidad, sino además las emergentes, incluso las latentes. Industria argentina. La fábrica es para los que trabajan, la película del director Ricardo Díaz Iacoponi, muestra esto en alguna medida. El fenómeno del surgimiento de las empresas recuperadas y la constitución de cooperativas de trabajadores es abordado a través de su argumento. Las angustias de un grupo de obreros, afectados por la crisis argentina, aparecen en sus diferentes escenas junto a las esperanzas que van forjando en el camino de defender sus trabajos y asumir el desafío que supone la autogestión obrera. Sin embargo, la película evidencia también con sutiliza el peso que los saberes de los trabajadores poseen en los 
procesos de producción y la desvalorización que sufren cuando son afectados por el despido y el desempleo. Así como los nuevos saberes que van forjando en la experiencia emprendida.

¿La producción de los saberes generados por las y los trabajadores, en el marco de las experiencias de economía social y solidaria, ${ }^{1}$ constituye una variable determinante de los procesos de desenvolvimiento de las mismas?

Los saberes desenvueltos desde las diversas experiencias generadas desde la economía social y solidaria por las y los trabajadores - como las presentadas en la película de Díaz Iacoponino solo constituyen un recurso al cual apelar para persistir en la forja de una manera diferente de organizar el mundo del trabajo, sino también es un elemento clave de su identidad individual y colectiva, un insumo para resistir frente al proceso de marginalización de la fuerza de trabajo generado por el capital y una fuente potencial e innegable de innovación de los procesos de producción económico social y solidario. Sin embargo, este conjunto de características asignadas a estos saberes, así como ellos mismos, no son reconocidos en su mayoría y ubicados, desde un canon epistemológico académico universitario eurocéntrico, en un nivel inferior.

¿Esta perspectiva respecto al saber producido, desde las experiencias de economía social y solidaria, es hegemónica? ¿No existen otras perspectivas que disputen con ella o la confronten? ¿La universidad mantiene inalterable el canon epistemológico académico universitario de carácter eurocéntrico? ¿Existen experiencias que muestren evidencias de vinculaciones diferentes entre universidad y economía social y solidaria en Latinoamérica?

Queremos centrar este ejercicio exploratorio en el examen de dos casos: El Programa de extensión universitaria Construyendo redes emprendedoras en Economía social (CREES) de la Universidad Nacional de Quilmes, del Gran Buenos Aires, Argentina; y el Seminario de Economía social, solidaria y popular de la Universidad Nacional Mayor de San Marcos, de la ciudad Lima, Perú.

Nos centraremos en casos tomados de las realidades de Argentina y Perú por la accesibilidad a la información que disponemos y porque estos países representan polos opuestos en lo referido al fomento de la economía social y solidaria desde las universidades: Argentina, con políticas públicas de fomento y promoción de la misma con participación de universidades; y Perú, con un casi nulo interés desde el Estado por ella y vínculos restringidos entre ésta y las universidades. No pretendemos, arrogantemente, agotar una discusión, sino más bien abrir una cuestión que creemos es central hoy para sociedades como las latinoamericanas, urgidas por ampliar y profundizar los procesos de democratización de la democracia, incluidos los referidos a los modos de producción de saber y conocimiento.

\section{UNIVERSIDAD Y ECONOMÍA SOCIAL Y SOLIDARIA}

El abordaje de la discusión sobre las relaciones entre universidad y economía social y solidaria no es nuevo en la reflexión latinoamericana. Uno de los que propone su tratamiento a comienzos del siglo xxi es Coraggio. Su crítica al economicismo-utilitarismo, característico del planteamiento neoliberal, sustenta una lectura alternativa de las universidades y sus relaciones con la sociedad y la economía. Él señala al respecto, cuando aborda la relación entre las universidades y lo local: «[...] implica tener una propuesta de universidad que entre en confrontación 
directa con el programa de su disolución a manos del mercado.» (Coraggio, 2002, p. 10). ${ }^{2} \mathrm{El}$ mismo autor (Coraggio, 2014), en otro texto escrito años después, precisa sobre la relación entre economía social y solidaria y universidad:

La perspectiva de una economía social y solidaria da lugar a un programa de acción estratégica para la transición, basado en prácticas micro, meso y sistémicas con dimensiones sociales, culturales y políticas, que requiere la transformación de una serie de instituciones que una mirada economicista afirmaría que no son parte del sistema económico (de mercado) a menos que justamente pauten la organización económica como negocios privados [...] Una de esas instituciones es la universidad, una forma histórica de pautar la producción y distribución del conocimiento «racional», como activo y como potencialidad, en ambos casos de fuerte y creciente efectividad en el desempeño de las economías. De hecho, las universidades y centros de investigación han estado traccionadas durante mucho tiempo hacia la demanda (sostenida pecuniariamente) de los grandes negocios y el Estado neoliberal tanto en cuanto a la formación de profesionales como a la investigación sobre problemas que definen como prioritarios, porque obstaculizan el crecimiento de capitales privados o de la economía nacional. (p. 4)

Una revolución desde la perspectiva de la economía social y solidaria en el sistema universitario - en la formación, la investigación, los servicios y la acción conjunta con la comunidad como el propio Coraggio anota-, implica revisar características fundantes de la modernidad y de la cual la universidad es heredera. Esto supone abrirse a cuestiones como el dejar de reproducir y sostener a pesar de sus fracasos el paradigma del desarrollo y la modernización dando cabida a la descolonialidad del poder y el saber. ${ }^{3}$

Sin embargo, Coraggio - en el texto anteriormente citado - también comparte una evaluación crítica de las relaciones establecidas entre los universitarios y la universidad con los actores de la economía social y solidaria. Éstas han sido — según su propio planteamiento«limitadas a intervenciones marginales y orientadas moralmente (extensión universitaria, mayormente estudiantes socialmente comprometidos, con tendencias al paternalismo)». Agrega además que «también se atienden las demandas mediadas por contratos públicos de servicios de formación e investigación, en buena medida dirigidas al diseño, gestión y evaluación de políticas sociales asistencialistas focalizadas en la pobreza extrema» (Coraggio, 2014, p. 5).

Esta crítica nos parece aguda, pero al mismo tiempo provocadora. No podemos dejar de mencionar, en primer lugar, que como institución la universidad latinoamericana posee una historia antigua, y en más de un caso antecede al propio Estado, por lo tanto, su tratamiento no debería ser simplificado. Todo lo contrario, constituye un viejo teatro, un punto de encuentro entre actores diversos, de procesos y tendencias determinadas desde libretos escritos muchas veces desde fuera o más allá de la universidad, enmarcado en estructuras no solo ancladas localmente, sino además nacional y hasta globalmente.

Lo segundo es que la economía social y solidaria tampoco constituye un sector pasivo o caracterizado por reunir actores sin iniciativa o desmotivados de relacionarse con otros actores y construir sus propios derroteros. Todo lo contrario, el dinamismo de los procesos que vienen desenvolviéndose desde la economía social y solidaria es intenso y abre varias posibilidades de relaciones en diferentes planos; incluida la posibilidad de nuevas perspectivas en los vínculos tejidos con la universidad y la sociedad. 
El supuesto sobre el cual partimos es que la universidad no responde solo a una determinación externa provocada por decisiones de política tomadas desde el Estado o a la imposición de intereses desde la sociedad y el mercado, sino además es resultado de la acción o inacción de los actores universitarios: autoridades, docentes, juventudes universitarias, trabajadores no docentes, graduados además de un contexto de acelerados cambios globales que suponen nuevas relaciones y ámbitos de acción para la universidad; así también como de reconfiguración de los actores con los cuales la universidad, en mayor o menor medida, se relaciona y, por lo tanto, sus expectativas, demandas reales o potenciales, reivindicaciones programáticas o agendas, experimentan cambios profundos y generan nuevos desafíos para ella. ${ }^{4}$ ¿La universidad, entonces, es realmente un punto de encuentro con la economía social y solidaria? Apelando una vez más a Coraggio (2014), podemos señalar:

Un claro ejemplo de la inadecuación e inflexibilidad del sistema universitario respecto a las demandas directamente sociales, es el reciente recurso de los movimientos indígenas a crear sus propias instituciones de formación e investigación, aún por fuera del sistema de reconocimiento formal de las capacidades de los que participen de esos procesos. A la vez, los profesionales para el sector público no son formados para establecer una relación dialógica entre saberes con los sujeto-objetos de las intervenciones estatales, reproduciendo la cultura de «expertos» propia de la Modernidad, hoy bajo crítica. (p. 5)

Por lo tanto, desde su argumentación, las posibilidades de que las universidades puedan convertirse de manera efectiva en lugares de encuentro con la economía social y solidaria son restringidas. A pesar de ello, la propia argumentación de Coraggio (2014) permite la posibilidad de encontrar un elemento clave que coadyuve a revertir esta situación. Él señala al respecto: «Es preciso que haya sujetos colectivos comunitarios y sociales que planteen sus necesidades de formación, conocimiento y servicios en una relación dialógica institucionalizada pero abierta a la innovación y el aprendizaje. Esto puede permearse al conjunto de la universidad que, sin embargo usualmente se resistirá burocráticamente». (p. 5).

Entonces, no está cerrada del todo la posibilidad de convertir a la universidad en un lugar de encuentro con la economía social y solidaria. Lo interesante, desde nuestro punto de vista, es que estamos otra vez confrontando con un planteamiento que recupera el peso de los actores, pero, en este caso, pensados desde fuera de la universidad. Si antes llamamos la atención sobre la necesidad de reconocer a los actores de la universidad, desde este planteamiento se advierte sobre la urgencia de reconocer en la sociedad a los actores que potencialmente puedan interpelarla y provocar nuevos procesos que redefinan sus vínculos. Este abordaje nos parece mucho más conveniente metodológicamente para el tratamiento de las relaciones entre universidad y economía social y solidaria, porque nos exige identificar y analizar el funcionamiento de los actores, indagar en las relaciones que desenvuelven, comprender sus discursos, recuperar sus memorias, antes que presuponer que las estructuras económicas, sociales, culturales o políticas constituidas los determinan inexorablemente.

No es que desconozcamos el peso que las estructuras poseen, sobre todo en el caso de dinámicas institucionales como las desenvueltas desde la universidad, pero es necesario ir más allá de los condicionamientos estructurales y avanzar en una perspectiva estructural 
histórica que pueda incluir a los actores que forjan las historias de la economía social y solidaria y la universidad.

Un aspecto necesario de sumar a la discusión, y que adquiere particular transcendencia, tiene que ver con lo que Germaná llama «el carácter monocultural de las universidades» y el imperativo de que se conviertan en interculturales. Lo intercultural requiere espacios «donde dialoguen en igualdad de condiciones las diversas tradiciones culturales, tanto las que han sido hegemónicas en el patrón de dominación moderno/colonial, como las perspectivas de conocimiento que habían sido reprimidas o excluidas por este patrón de dominación social. Se trata del principio de la «ecología de saberes» que propone Boaventura de Sousa Santos, el que «consiste en la promoción de diálogos entre el saber científico y humanístico que la universidad produce y los saberes legos, populares, tradicionales, urbanos, campesinos, provincianos, de culturas no occidentales (indígenas de origen africano, oriental, etc.) que circulan en la sociedad (citado por Germaná)». (Germaná, 2013, p. 17).

La universidad latinoamericana posee predominantemente un carácter monocultural, porque busca en su mayoría reproducir el patrón de dominación moderno colonial eurocéntrico; pero afronta tensiones profundas, caracterizada por la heterogeneidad estructural y cultural de su sociedad. ${ }^{5}$ La universidad actúa muchas veces como enclave que recluta jóvenes que adquieren la condición de universitarios a través de un proceso de formación que en general los aleja de sus comunidades y lugares de origen. O contrapone los saberes que portan y heredan de sus familias y comunidades con los saberes de las comunidades científicas existentes dentro de la universidad, estableciendo jerarquías con base en modelos y estereotipos foráneos, provocando su desvinculación y desarraigo con los lugares donde nacieron, reproduciendo mecanismos de dependencia cultural, científica y tecnológica. Este conjunto de elementos entran en contraposición con la demanda de reconocimiento creciente que reclaman las varias tradiciones culturales, y los saberes que poseen y reproducen las sociedades latinoamericanas.

La vinculación de la universidad con la economía social y solidaria es un paso para enfrentar esto. Demostrarlo constituye una tarea urgente para confirmar que la universidad, además de ser un viejo teatro que puede servir como punto de encuentro con la economía social y solidaria, puede ser refundada sobre la base de la capacidad de los actores universitarios para abrirse al diálogo y reconocimiento de procesos y actores nuevos.

\section{EXPERIENCIAS Y ACTORES UNIVERSITARIOS Y SOCIALES}

Es pertinente empezar señalando que las experiencias de vinculación entre economía social y solidaria y universidad no son recientes en Latinoamérica, e incluso reúnen en algunos países una historia larga, como podría ejemplificarse en el caso de Venezuela con relaciones entre cooperativas y universidad que, como la Universidad de Los Andes de Mérida, posee antecedentes que se remontan por lo menos a los años sesenta. Estas relaciones son desenvueltas desde la creación del Centro de Estudios Cooperativos, fundado en 1963, como indica García (2007, p. 17). México, por su parte, posee el caso de la Universidad Autónoma de Querétaro que desde 1969 forma técnicos en cooperativas; enseñanza que eleva a carrera universitaria, desde el 2009, bajo el nombre de Gestor de empresas sociales, como señala Izquierdo (2009, p. 116). 
Estas vinculaciones no solo incluyen a las cooperativas, o a las organizaciones de la economía social tradicional o histórica, sino también a experiencias más recientes como las desenvueltas a partir de las empresas recuperadas que, como en el caso de Brasil, han establecido relaciones con la universidad, como la Universidad Federal de Río de Janeiro, la primera en tomar la iniciativa a través de las llamadas Incubadoras Tecnológicas de Cooperativas Populares (ITCP), creadas desde mediados de los años noventa y que a la fecha suman setenta experiencias en todo el país, como han evidenciado Cruz, Tillman, Della Vechia y Nunes (2011). ${ }^{6}$

Los casos específicos de Argentina y Perú permiten también registrar evidencias de vínculos entre economía social y solidaria y universidad desde varias décadas atrás. El caso argentino muestra que si bien la mayor parte de la oferta de formación actualmente existente en economía social y solidaria, así como en cooperativismo, surge después del año dos mil nueve carreras de grado y diecisiete de posgrado-, existió un programa de formación universitario en cooperativismo que otorgaba una licenciatura en la Universidad Nacional de La Plata entre 1953 y 2002, el primero de Latinoamérica, la cual luego es convertida en tecnicatura, como anotan Acosta y Levin (2013, p. 7). Sin embargo, es pertinente anotar que a pesar de la existencia de esta importante oferta de formación se observa - como las mismas autoras señalan - una escasa articulación tanto entre las diversas carreras o unidades académicas en donde se dictan entre sí como entre la universidad y las empresas cooperativas y los órganos de representación - federaciones y confederaciones- (2013, p. 15).

El caso peruano es diferente del argentino porque el tema de la economía social y solidaria no es visible ni mucho menos central de la agenda pública, no debemos olvidar el contexto de aplicación de políticas de liberalización de mercados, caracterizado por ser particularmente duro e inflexible, desplegado desde comienzos de los años noventa, de la mano con la implementación de un régimen autoritario como el liderado por el presidente Fujimori, que llevó a la legitimación de un discurso extremo promercado sin atenuantes en el conjunto de la sociedad peruana. Sin embargo, es posible registrar antecedentes de los vínculos tejidos entre economía social y solidaria y universidad, particularmente con las cooperativas, desde los años sesenta. Por lo menos, en dos universidades: la Universidad Particular San Martin de Porres y la Universidad Nacional Federico Villareal, existieron programas dirigidos a la formación profesional en cooperativismo y actividades de extensión universitaria con cooperativas, desde 1966, como indica Cárdenas (1983, pp. 356 y 359).

Lo paradójico es que en paralelo al contexto generado por las políticas de liberalización de mercados se produjo lo que Mogrovejo, Vanhuynegem y Vásquez llaman el «renacimiento del modelo cooperativista peruano», caracterizado por el resurgimiento de un amplio y diverso número de experiencias cooperativistas, especialmente, desde el sector agrario vinculado al mercado global, dedicado a la exportación de productos como el café; pero donde, por un lado, la discusión sobre la noción de economía social y solidaria está presente gracias a determinados agentes - iglesia, organismos no gubernamentales, cooperación internacionalque se constituyen en sus principales difusores y, por otro lado, la vinculación al mercado global es desenvuelto en gran medida gracias a las redes de comercio justo. No podemos dejar de mencionar, como referencia, que la mitad del valor total de las exportaciones de café peruano proviene del comercio justo (Remy, 2007, p. 49).? 
Por ello, los pocos esfuerzos que hubo para vincular economía social y solidaria, principalmente cooperativas, y universidades, sobre todo en el campo de la formación y la extensión universitaria, fueron debilitados o desaparecieron en el marco del contexto adverso de aplicación de las políticas de liberalización de mercados. Hoy existe solo un posgrado universitario en el conjunto de la oferta de formación de las universidades peruanas dirigido al otorgamiento de una diplomatura en gestión y administración de cooperativas, brindada por la Facultad de Ingeniería Administrativa e Industrial de la Universidad Inca Garcilaso de la Vega en coordinación con la Confederación Nacional de Cooperativas del Perú.

Es necesario mencionar además de manera complementaría que en la última década se desarrolló una oferta de programas de formación brindada desde los propios movimientos de la economía social y solidaria y las cooperativas - de ahorro y crédito, de servicios, trabajadores - que renacían y animaban esta oferta, pero desvinculada de las universidades, de manera dispersa, fragmentada, carente de continuidad, permanencia en el tiempo e identidad, que conlleva a una constante fuga de cuadros técnicos hacia otras modalidades empresariales. Lo que puede ser sintetizado en la declaración de un viejo activista cooperativista cafetalero y actual gerente de la Junta Nacional del Café, Lorenzo Castillo: «Hoy las universidades forman gerentes para negocios urbanos, no para empresas rurales y mucho menos para cooperativas». (Mogrovejo, Vanhuynegem y Vásquez, 2012, p. 338). Las particularidades de los dos casos que examinaremos nos permitirán entrar en detalle, respecto a los vínculos entre economía social y solidaria y universidad, en realidades como las de Latinoamérica.

\section{PROGRAMA DE EXTENSIÓN UNIVERSITARIA CONSTRUYENDO REDES EMPRENDEDORAS}

EN ECONOMÍA SOCIAL DE LA UNIVERSIDAD NACIONAL DE QUILMES

La Universidad Nacional de Quilmes (UNQ) está ubicada en el partido o municipio del mismo nombre, constituye uno de los 135 municipios que integran la provincia de Buenos Aires y es uno de los más antiguos del conurbano, ubicado en el sudeste, sobre la costa del Río de la Plata, a $17 \mathrm{~km}$ de la capital federal. ${ }^{8}$ El Partido de Quilmes ha sido uno de los más afectados por el proceso de crisis experimentado como resultado del agotamiento del modelo neoliberal desde mediados de los noventa, y por ello es afectado por el incremento de la pobreza y el desempleo, citando a Fidel, Di Tomaso y Farias (2008):

La trama urbana del Partido - se comienza a ocupar de gente con hambre, buscando entre la basura y la mendicidad su sustento; aparece, hasta esos momentos desconocidas, nuevas modalidades de violencia social; emerge el desencanto por los políticos y sus actividades: comienza a exhibirse un entorno de fábricas vacías de contenido y sentido; simultáneamente, también surgen nuevas búsquedas y formas de asociación y movimientos sociales urbanos, acercamiento que se cimienta en nuevos lazos sociales entre los vecinos, por ejemplo en el Partido de Quilmes, usando las instalaciones de una fábrica vacía, a mediados de los noventa, se crea el núcleo central de un Club de Trueque, lugar de intercambio de productos que utiliza monedas producidas por ellos mismos, y que básicamente es un marco de contención afectiva y material. Dicho modelo de asociación, en los inicios del año 200o, entre otras razones por cuestiones organizativas y por efecto de los precios externos a la esfera del propio Club de Trueque, comenzó a transitar hacia su crisis final. (p. 38) 
La UNQ forma parte de este contexto territorial particularmente afectado por la agudización de procesos de exclusión y marginalización resultantes de la crisis argentina de la época. Su creación el 23 de octubre de 1989, por Ley N. ${ }^{\circ} 23749$ del Congreso de la Nación y su normalización el 12 de diciembre de 1992, no son hechos que hayan pasado inadvertidos para las poblaciones de este partido. El hecho de que el edificio de la universidad fuera construido sobre terrenos donados por la empresa textil Fabril Financiera y abriera sus puertas con solo diez aulas para sus primeros mil alumnos, constituyen elementos simbólicos claves que enraízan de manera particularmente sentida a esta institución académica con su territorio y su comunidad.

Es necesario anotar, además, que la UNQ está inscrita en otro proceso igual de determinante, como los antes mencionados: el despliegue de un conjunto amplio y diverso de experiencias, desde por lo menos comienzos del 2000, orientadas por la convicción de que, como dice Rofman (2005), un nuevo modelo de vinculación entre las instituciones académicas y su entorno social está generándose. En el contexto de crisis institucional vivido en la Argentina a fines del 2001 destaca el prestigio y la legitimidad de las universidades, atributos que les permiten asumir un rol de liderazgo o de mediación en el proceso de articulación multiactoral y desenvolver procesos de participación, acciones a nivel local, experiencias de involucramiento activo con la comunidad, bajo la forma de intervenciones sostenidas en el aporte de conocimiento, información o metodologías, relativo al proyecto en el que la universidad está implicada (p. 25).

El reconocimiento de este proceso previo, de despliegue de experiencias donde las universidades juegan un rol central en el contexto de la crisis argentina de comienzos del 2000 , constituye una variable importante a tomar en cuenta, porque contextualiza de manera adecuada el esfuerzo realizado desde la UNQ, pero además porque proporciona la posibilidad de comprender otras experiencias de vinculación entre economía social y solidaria y universidad, desplegadas con sus propias singularidades y asumir su experiencia como parte de un proceso y no como algo aislado.

La experiencia de la UNQ surge en el 2006 — como señalan Sena, Baliño y Martínez (2006)— desde iniciativas desenvueltas por docentes del Departamento de Economía y Administración. ${ }^{9}$ Parte del impulso de propuestas para el fortalecimiento y desarrollo de la economía social y solidaria desde esta universidad. Trabaja de manera articulada en proyectos de extensión, investigación y formación con destacados desempeños y resultados, como el Diploma de Operador Socioeducativo en Economía Social y Solidaria (DOSESS), promoviendo la formación en el territorio y prácticas educativas solidarias de estudiantes, graduados y docentes de la UNQ, en servicios comunitarios vinculados a sus recorridos académicos. Sena, Baliño y Martínez (2006) indican: «La experiencia tiene tres objetivos: impulsar prácticas universitarias de aprendizaje y servicio solidario que fortalezcan los procesos de gestión asociada y redes organizacionales en Economía Social y Solidaria, promover prácticas universitarias solidarias que impulsen el desarrollo de experiencias de cooperación social y favorecer la formación de agentes, promotores y dirigentes en Economía Social y Solidaria». (p. 170).

El planteamiento teórico de soporte usado en la experiencia es la noción de economía social y solidaria. Definido como un campo plural — desde la reflexión de Pastore (2010), uno de los principales animadores de la experiencia- y referido a las experiencias históricas del 
cooperativismo y mutualismo — conjunto también llamado «economía social tradicional»—, así como a las nuevas formas organizativas de hacer economía social - llamada «nueva economía social» o «economía solidaria»- Conjunto que abarca una diversidad de experiencias, organizaciones y emprendimientos que tienen características distintivas entre sí, pero que poseen una matriz identitaria de atributos compartidos, entre los que se destaca el desarrollar actividades económicas con una definida finalidad social —en términos generales, mejoramiento de las condiciones, ambiente y calidad de vida de sus propios miembros, de algún sector de la sociedad o de la comunidad en un sentido más amplio-, a la vez que de carácter asociativo y gestión democrática en un contexto de autonomía tanto del sector privado lucrativo como del Estado (Pastore, 2010, p. 2).

Una de las acciones principales que se implementará desde la experiencia será el Dosess, el que en su primera etapa, apelando otra vez a Sena, Baliño y Martínez (2012), se vinculó al Programa Argentina Trabaja de manera articulada con el Ministerio de Desarrollo Social de la Nación, Ministerio de Educación de la Nación y la UNQ, desde 2010 y 2011, capacitando a 800 cooperativistas distribuidos en veinticinco sedes del Gran Buenos Aires, emplazadas en universidades públicas e institutos de formación superior. El diploma fue una respuesta institucional hacia una creciente demanda social de capacitación operativa integral surgida de las propias experiencias de trabajo autogestivo y de las políticas públicas de fomento y apoyo a la economía social (p. 171).

Un aspecto no resaltado por los autores pero que puede inferirse de la experiencia realizada es el enraizamiento que esta institución académica logra en el marco de la experiencia que anima con su territorio y comunidad, el reconocimiento del proceso de aprendizaje que logra de la gente con la cual trabaja y dota de sentido la labor de la universidad y las y los universitarios, como parte de un proyecto de sociedad con fuerte presencia del Estado. Algo que en alguna medida puede apreciarse en las palabras sentidas de Antonia Aquino, una ciudadana de Avellaneda, capacitada gracias a esta experiencia y cuyas declaraciones son citadas por Sena, Baliño y Martínez: «La Diplomatura lo que hace es educarnos para lo que viene. Estar formándonos de esta manera es algo que nos va a quedar. Y eso es un Estado presente, que te incluye, te enseña y te da herramientas de trabajo. Para que, si mañana no está, nosotros con lo que aprendimos sepamos construir otras herramientas, otras formas de trabajo. Es un Estado que nos forma y nos educa, para enfrentar otras situaciones». (2012, p. 174).

$\mathrm{Al}$ respecto el trabajo de Pastore, Altschuler, Schmalko y Zeballos (2011) - anterior al de Sena, Baliño y Martínez- anota algunos elementos que consideramos importantes de sumar a la reflexión: la experiencia es desplegada en el conjunto del territorio del conurbano, de la ciudad de Buenos Aires, en alianza o red con otras cinco universidades públicas; la relación con lo político, fuera de la universidad, es fuerte por la presencia de figuras como Alicia Kirchner, ministra de desarrollo social; la iniciativa y la experiencia del Dosess contribuyó para incidir en las autoridades de la UNQ y conseguir la aprobación de la Tecnicatura Universitaria en Economía Social y Solidaria (TUESs); la relación de la experiencia con los movimientos sociales es creciente y puede confirmarse, entre otras acciones, con la firma de convenios como el establecido con la Confederación Nacional de Cooperativas de Trabajo (CNCT) (2011, pp. 12-14). 
Finalmente, en términos de perspectivas de futuro, la experiencia tiene considerada la implementación de un proceso de investigación y sistematización que es asumido como fundamental para proveerse de nuevas herramientas analíticas, pedagógicas y organizativas para la profundización y mejora constante de la experiencia de extensión (2011, p. 14); es decir, la labor de investigación es concebida desde una perspectiva de investigación participativa o investigación acción y no estrictamente respondiendo al canon académico alejado del diálogo de saberes. La investigación además es articulada de manera directa a las labores de extensión universitaria y a las de formación y docencia; no es desenvuelta, por lo tanto, independientemente, sino articulada al proceso de despliegue de la experiencia; más aún si bien no es mencionado por el autor y las autoras es evidente que este proceso de investigación supone una dinámica multi, inter y transdiciplinaria, por las mismas exigencias que supone y que rompen en la práctica con la compartamentalización del conocimiento y los campos disciplinarios.

Una conclusión derivada de la experiencia — siguiendo una vez más a Pastore, Altschuler, Schmalko y Zeballos- es que es necesario resaltar la productividad y riqueza de la sinergia producida entre los proyectos de extensión universitaria desarrollados, que permiten vincular estrechamente experiencias concretas de economía social y solidaria y políticas públicas orientadas a la promoción del sector, con la extensión universitaria y la labor de formación y docencia desenvuelta desde la universidad (2011, p. 14).

¿Qué aspectos pueden ser reconocidos como necesarios de afianzar a futuro en la experiencia y son ineludibles de considerar? Señalamos dos, uno al interno, la relación con la formación de grado y posgrado; y otro al externo, la relación con el Estado.

La relación con la formación de grado al interno es, sin lugar a dudas, un aspecto clave sobre el cual la experiencia debe prestar atención. El peso puesto en la extensión universitaria y las relaciones hacia fuera de la universidad constituyen componentes estratégicos, pero la relación al interno sobre todo con la formación de grado no muestra el mismo nivel de despliegue y afianzamiento. Incluso puede reconocerse como una debilidad en la medida que no logra vincularse de manera consistente con la dinámica de los tres departamentos de la universidad - economía y administración, ciencias sociales, ciencias y tecnología- y con las 35 carreras que oferta. No es que deba relacionarse necesariamente con todos, el hecho es que resulta más que evidente que al no hacerlo por lo menos con una parte, la experiencia aparece como un proceso muy interesante; pero externo a la dinámica misma de la universidad, que pasa por la actividad docente animada desde los departamentos o los cursos impartidos en las carreras y a través de los cuales las y los estudiantes son entrenados y viven el día a día de la universidad, por mencionar un par de ejemplos.

La creación de la Tecnicatura Universitaria en Economía Social y Solidaria cubre en parte esto, sin embargo, afianza más el vínculo hacia fuera, sobre todo con los activistas de los actores de las economías sociales y no logra del todo generar ese eslabonamiento que nos parece clave provocar con la actividad de departamentos y carreras. Tampoco establece una relación con la oferta de posgrado - 3 doctorados y 12 maestrías - e igualmente desenvuelve una relación externa y paralela a la actividad de formación e investigación ahí desenvuelta. Cubrir este aspecto sería crucial en la medida que el entrenamiento desplegado podría beneficiarse y beneficiar a la vez a la experiencia. 
La articulación con el grado y el posgrado son aspectos claves. El reto es hilvanar una propuesta que enhebre formación, investigación y extensión universitaria, en una estrategia que supere la compartamentalización o el aparente paralelismo en el que podría percibirse cae la extensión universitaria, a fin de integrarlas y complementarlas, hacer que dialoguen retroalimentándose creativamente. La relación con el Estado aparece como otro asunto crítico. El nivel de relación establecido con políticas y programas estatales, como Argentina Trabaja, es una muestra evidente de una apuesta política legítima de asumir, pero también provoca la interrogante respecto a los niveles de autonomía de la experiencia y sobre su capacidad de resistir respecto a la imposición de posibles formas de corporativismo estatal.

Surge además, de la mano con esta preocupación, la pregunta respecto al rol cumplido desde la experiencia frente a posibles conflictos, normales que surjan como parte de la dinámica social, entre los actores de la economía social y solidaria y el Estado. Anotemos que estos tienen la característica de ser sobre todo, aunque no únicamente, actores urbano populares localizados. El fuerte anclaje territorial de la UNQ determinó en gran medida el establecimiento de relaciones con los actores propios de su entorno. No es complicado deducir que asegurar la autonomía de la experiencia es determinante para el despliegue de un proceso que priorice las necesidades y demandas de estos actores antes que los intereses políticos existentes desde el Estado.

Este asunto tal vez es uno de los más delicados a examinar y exige la búsqueda de estrategias que aporten al afianzamiento de la autonomía de la experiencia o a una gestión que tenga la capacidad de afrontar los conflictos y tensiones en su relación con el Estado, libre de posibles sometimientos a formas de imposición, sutiles o manifiestas, de corporativismo estatal.

\section{SEMINARIO DE ECONOMÍA SOCIAL, SOLIDARIA Y POPULAR DE LA}

UNIVERSIDAD NACIONAL MAYOR DE SAN MARCOS

La Universidad Nacional Mayor de San Marcos (UnMsm) fue fundada el 12 de mayo de 1551, posee actualmente 20 facultades y 62 carreras profesionales de grado, 28 doctorados y 96 maestrías, fuera de diplomaturas y segundas especializaciones. Tiene, según información de la Asamblea Nacional de Rectores (2011), una población de 28.645 estudiantes de grado, 53.108 postulantes en promedio compiten cada año por 5781 vacantes.

La UNMSM no puede ocultar los problemas que padece como resultado de un largo proceso de crisis acumulada. Al respecto, López (2013) señala:

La masificación y abandono [por parte del Estado] de [la mayor parte de] las universidades públicas generaron la mediocrización de la formación académica y profesional Aquello incrementó, por el lado de la demanda, la dificultad para incorporarse al mercado de trabajo. Sobre la base de la masificación, la mediocrización y el abandono estatal, la autonomía universitaria se volvió autarquía y el cogobierno devino en anarquía creando un clima propicio para la frustración estudiantil y para la emergencia de ideologías de la desesperanza que culminan en el terrorismo. El movimiento estudiantil se diluyó y fragmentó, la calidad académica decayó, los operadores aparecieron en la escena, la corrupción se instaló en la universidad y las autoridades universitarias buscaron perpetuarse en el poder a través de métodos clientelares y mafiosos. (p. 17) 
Este contexto de crisis característico de la universidad del Perú permite comprender de manera más integral el entorno de la experiencia que analizaremos. A diferencia del caso de la UNQ estamos frente a una institución universitaria pública de mayor tamaño y antigüedad. Sumado al hecho, como indicamos antes, que en el Perú el tema de la economía social y solidaria no es un tema visible ni mucho menos central de la agenda pública. La experiencia del Seminario de Economía Social, Solidaria y Popular de la UNMSM surge el 2012, desde la Facultad de Ciencias Sociales, ${ }^{10}$ "con motivaciones y apuestas individuales de docentes de esta facultad, que convergen y dan pie a un proceso de institucionalización en la universidad», como indica Pérez (2014, p. 2).

El Seminario - según la Resolución Rectoral N. ${ }^{\circ}$ 03998-R-14- constituye un grupo de investigación acción de tipo permanente, que congrega a docentes, estudiantes y representantes de movimientos sociales, orientado por tres objetivos: reflexionar y producir conocimientos sobre la problemática de la economía social, solidaria, popular; formar y entrenar en el manejo de instrumentos teóricos y metodológicos relacionados con procesos de intervención; apoyar el fortalecimiento de experiencias de gestión local con protagonismo de organizaciones sociales, culturales, económicas e instituciones públicas y privadas.

El planteamiento teórico de la experiencia fue nutrido desde dos vertientes: por un lado, una perspectiva ecléctica en la medida que reconocía corrientes de pensamiento diversas como parte de un proceso amplio de discusión e indagación sobre la problemática de la economía social y solidaria o y el Buen Vivir, preocupada por el recojo de evidencias respecto a las relaciones económico sociales, culturales, políticas, con la naturaleza, desenvueltas por cooperativas, comunidades y pueblos originarios, redes de productores de comercio justo y agroecológicos, asociaciones de artesanos, bancos comunales, comedores populares autogestionarios, entre otros.

De otro, el aporte histórico estructural trabajado desde las reflexiones pioneras de Quijano sobre el "polo marginal», realizadas a fines de los años sesenta. Su planteamiento asumió que Marx registró las tendencias centrales de la problemática de la sobrepoblación relativa o ejército industrial de reserva; pero que estas adquirían contemporáneamente mayores niveles de contradicción. El término «marginalidad» en el planteamiento de Quijano se refiere, en este sentido, a una nueva dimensión de la problemática de la sobrepoblación relativa (Quijano, 1977, p. 19). Esta noción surge de una perspectiva de interpretación crítica del proceso de desenvolvimiento capitalista latinoamericano, porque lo que está en el centro de su planteamiento es qué ocurre con la fuerza de trabajo y los trabajadores que no logran vender su fuerza de trabajo al capital, y especialmente, qué actividades emprenden, cómo las organizan, y también qué territorios las albergan. No es difícil deducir que su reflexión va más allá de la crítica al crecimiento económico y la modernización capitalista, porque indaga en las bases mismas de constitución del capitalismo latinoamericano, en las relaciones que lo sustentan, y demuestra que sus posibilidades reales de crecimiento y aporte a la modernización de la sociedad tienen debilidades estructurales innatas que lo limitan. ${ }^{11}$

El seminario fue constituido desde el Comité Asesor de la Escuela Académico Profesional de Sociología. ${ }^{12}$ Es pertinente anotar que un argumento determinante para convencer a los integrantes del comité asesor fue la propuesta de establecer alianzas con otras instituciones 
académicas, a fin de generar una institucionalidad de soporte y animar procesos de investigación acción que contribuyeran a su posicionamiento, así como a revertir su aislamiento. Este aspecto es particularmente sentido por el nivel de desarticulación institucional que la Facultad de Ciencias Sociales, y sus diferentes escuelas y departamentos, afronta actualmente por la crisis que la afecta.

La presentación de un convenio con la Universidad del País Vasco que, a través de Hegoa Instituto de Estudios sobre Desarrollo y Cooperación Internacional, había manifestado su interés de iniciar un trabajo con la UNMSM, terminó de convencer al Comité Asesor para acordar la constitución del seminario. Sin embargo, la decisión de esta instancia debía pasar a un siguiente nivel en la jerarquía de la universidad: el Consejo de la Facultad de Ciencias Sociales, donde también debía recibir una aprobación.

En paralelo, el convenio con la Universidad del País Vasco fue elevado al rectorado y después de varias y largas gestiones, fue aprobado por Resolución Rectoral N. ${ }^{\circ}$ 03820-R-13 el 19 de agosto del 2013, pero la aprobación de la creación del seminario no tuvo igual resultado y demoró un largo periodo.

Un elemento extra es que los representantes de Hegoa, antes de la aprobación del convenio, plantearon un diálogo para evaluar el inicio de acciones conjuntas. El diálogo cedió paso al poco tiempo a la firma de un acuerdo de partes para avanzar en la presentación de un proyecto, a la Agencia Vasca de Cooperación para el Desarrollo, y cogestionar un conjunto de actividades a nivel de tres países andinos: Perú, Ecuador y Bolivia. La actividad principal planteada desde la UNMSM, en el marco de este proyecto, fue la implementación de un Observatorio de Economía Social, Solidaria y Popular, entendido como una herramienta de recojo de evidencias y aporte al proceso de fortalecimiento de organizaciones y movimientos sociales.

El 15 de noviembre de 2013 fue organizado un taller con representantes de organizaciones y movimientos sociales previamente identificados a través de un mapeo, y con los cuales fue desenvuelto un diálogo y reflexión colectiva sobre las relaciones entre universidad y organizaciones y movimientos sociales. Esta primera coordinación abrió un proceso que duró varios meses, el punto de partida fue desenvolver un diálogo de saberes y, por ello, la necesidad de conformar una instancia que permitiera este diálogo y rompiera con el aislamiento de la universidad respecto a la sociedad; pero además aportará a abrir la perspectiva de la propia universidad sobre los procesos de producción de saberes y conocimientos generados por organizaciones y movimientos sociales.

El 20 de marzo de 2014 en una ceremonia pública realizada en La Casona de la UNMSM, la sede histórica donde fue fundada esta universidad, fue firmada el acta de constitución del Consejo Social del Seminario y Observatorio de Economía Social, Solidaria y Popular; definido como «un órgano colegiado que cumple un rol de asesoría y facilitador del diálogo, la articulación y la cooperación entre la universidad y las organizaciones y movimientos sociales». El Consejo Social está integrado por varias de las principales centrales campesinas y de pueblos originarios del Perú, así como artesanos y productores de comercio justo y redes de economía social y solidaria, figuran: la Confederación Nacional Agraria, la Confederación Campesina del Perú, la Coordinadora Nacional de Pequeños Productores de Comercio Justo del Perú, la Central Interregional de Artesanos del Perú, la Federación Nacional de Mujeres 
Campesinas, Artesanas, Indígenas, Nativas y Asalariadas del Perú, la Central Café y Cacao, el Grupo Red de Economía Solidaria del Perú, el Grupo Género y Economía.

Este proceso permitió abrir una relación intensa y diversa con varias de estas organizaciones y movimientos, expresada en la implementación de prácticas preprofesionales, elaboración de reportes de investigación acción, diseño de planes estratégicos y formulación de proyectos de desarrollo, cursos en las carreras de la facultad, realización de audiovisuales, actividades de extensión universitaria y proyección social, organización de labores de voluntariado universitario, entre otras.

Paralelamente, a la conformación del Consejo Social, la labor de incidencia para lograr la aprobación del Consejo de Facultad de la creación del seminario fue continuado. El resultado de este proceso fue la Resolución de Decanato N. ${ }^{\circ}$ 0318-D-FCCss-2014 del 10 de abril de 2014, que aprueba la creación del seminario, resolución que meses después fue seguida por la Resolución Rectoral N. ${ }^{\circ}$ 03998-R-14 del 6 de agosto de 2014, ratificando su creación. Este largo proceso de incidencia interna y de vinculación con organizaciones y movimientos sociales fue complementado con la actividad principal del proyecto cogestionado: la implementación del observatorio. El resultado fue el diseño del portal web del observatorio - http://economiassolidarias.unmsm.edu.pe- $\mathrm{y}$ su alojamiento en el servidor de la universidad.

La experiencia del seminario, más allá de la descripción del proceso de incidencia desplegado dentro de la UNMSM, reúne desde nuestra perspectiva tres cuestiones necesarias de evaluar para el afianzamiento de la experiencia.

La primera cuestión es que muchas de las actividades realizadas desde el seminario con las organizaciones y movimientos no han logrado del todo basarse en el desenvolvimiento efectivo de un diálogo de saberes, porque en más de un caso son reproducidas jerarquías entre los saberes de la universidad y los de las organizaciones y movimientos. No es que no exista una apuesta clara por superar esto desde el seminario, todo lo contrario, la preocupación por superar el establecimiento de estas jerarquías es permanente y está presente. Sin embargo, la realidad es que se sigue reproduciendo desde el trato cotidiano hasta la labor misma desplegada como parte de la realización de las actividades. El hecho que estas actividades estén sometidas a través del Consejo Social a un mecanismo de evaluación permanente es uno de los pocos recursos al cual apelar para lograr de manera efectiva la superación de esta situación. El reto es afianzar este mecanismo de diálogo entre la universidad y las organizaciones y movimientos, a fin de fortalecer relaciones paritarias y evitar la reproducción de posibles asimetrías. El proceso de despliegue de un diálogo de saberes no constituye al final de cuentas una declaración de buenas intenciones, sino una práctica real nutrida de la vida social y, por lo tanto, de relaciones de poder necesarias de ser evidenciadas constantemente para afianzar un trato cotidiano más democrático.

La segunda cuestión es que el seminario financió sus actividades con fondos de cooperación internacional. El acceso a los mismos no ha sido amplio, pero sí ha representado un recurso clave para dar impulso inicial a su accionar; esto debido sobre todo a la crisis de la universidad que no le ha permitido acceder a financiamiento proporcionado por ella y no haber podido tampoco gestionar recursos provenientes de políticas y programas públicos o agentes y actores locales y nacionales. El establecimiento de alianzas estratégicas sur-norte constituyó en este sentido el medio principal al que apeló para desenvolver sus actividades. La interrogante que surge 
es hasta qué medida el seminario es dependiente de esta estrategia e incluso hasta dónde el uso y acceso a los recursos de la cooperación dejan de ser una estrategia o medio para convertirse en un fin en sí mismo. Sin lugar a dudas este asunto constituye uno de los desafíos mayores que tendrá para los próximos años; no tanto porque su acceso a los fondos de la cooperación internacional crezcan demasiado - a final de cuentas el Perú no constituye una prioridad actual de las financieras y agencias de cooperación internacional, lo que explica la reducción de los mismos en los últimos años-, sino porque la actividad del seminario puede hacerse dependiente de una sola fuente de recursos. Tal vez la combinación de fuentes de financiamiento es un aspecto clave a considerar, la presentación sostenida de proyectos a fondos de investigación de la propia universidad, por más limitados, exiguos o complicado que sea acceder a ellos, o la gestión ante fuentes de financiamiento público sobre todo dirigidos a ciencia y tecnología, deberían ser asuntos prioritarios desde un razonamiento interesado en preservar su autonomía económica y evitar la dependencia de los fondos de cooperación internacional.

Una tercera cuestión igual de importante tiene que ver con el afianzamiento de relaciones institucionalizadas hacia dentro de la universidad. Desde su inicio, el seminario ha intentado ligarse a los cursos de las carreras conducentes al grado y desde ahí construir procesos de producción de saberes o intervenciones aplicadas de la mano con actividades de docentes y estudiantes. Este nexo ha ligado su intervención a las actividades desenvueltas en departamentos y escuelas de la universidad. Esta relación hacia dentro y principalmente con los procesos de formación de grado ha contribuido a afianzar su entronque con el entrenamiento académico, porque anima además de labores de formación, tareas de investigación, así como experiencias de extensión universitaria y proyección social. No están del todo consolidadas estas relaciones, pero es evidente que su despliegue constituye un fuerte animador de actividades académicas.

La relación con el proceso de formación de posgrado constituye más bien un vacío y o una debilidad de la experiencia, al no haber logrado aún vincularse con su accionar. No es posible desconocer que los procesos de formación a nivel de posgrado constituyen un campo estratégico para la producción de saberes y la incidencia en la comunidad académica, necesario de trabajar a través de una oferta diversificada que contemple los estudios de doctorado, maestría y diplomados, dirigidos a posgraduados.

Tampoco ha sido posible aún desenvolver actividades que involucren a los trabajadores no docentes de la universidad. Han sido realizadas algunas actividades, principalmente ferias de productores y campañas de comercio justo que permitieron a los trabajadores no docentes participar sobre todo como consumidores; pero no ha sido tejido un nexo más sistemático para articular las labores del seminario a la actividad de este estamento de la comunidad universitaria.

\section{¿VINCULACIONES ENTRE UNIVERSIDAD Y ECONOMÍA SOCIAL Y SOLIDARIA?}

Las experiencias examinadas evidencian el desenvolvimiento de manera germinal, en ambas universidades, y con todas sus limitaciones, de esfuerzos encaminados a generar diálogos de saberes. No comparten el supuesto de desconocer los saberes producidos por organizaciones y movimientos sociales, invisibilizarlos o asignarles un nivel inferior en una supuesta jerarquía de conocimientos. 
No podemos afirmar evidentemente que desde las experiencias examinadas existe la decisión manifiesta por entrar a disputar o confrontar con la perspectiva hegemónica hoy vigente en muchas universidades, expresada en la desvinculación con la sociedad, las organizaciones y movimientos sociales y los saberes producidos desde sus prácticas. Tal vez incluso desde las experiencias consideradas no existe esta necesidad, porque su fundamento si bien tiene un soporte teórico metodológico crítico, no parte de la necesidad de resolver un problema solo y exclusivamente académico, sino más bien desplegar una experiencia viva, nutrida de relaciones sociales cargadas de un imaginario social sustentado en vinculaciones tejidas con organizaciones y movimientos sociales.

A pesar de ello, reconocemos que las experiencias analizadas muestran opciones diferentes, esfuerzos sostenidos y hasta caminos recorridos posibles de replicar en otras realidades, no necesariamente reproduciendo fielmente sus experiencias; pero sí recogiendo algunos de sus aprendizajes e incluso asumiendo algunas de las herramientas empleadas en sus procesos de autogestión y cogestión. Estas experiencias, por lo tanto, muestran que el canon epistemológico académico universitario de carácter eurocéntrico no es el único o exclusivo modelo de referencia para la producción de saberes y conocimientos. Las experiencias aportan prácticas teóricas aplicadas sustentadas en procesos dinámicos y de gran complejidad, no reducidas al ámbito de las dinámicas de las propias universidades, sino enhebradas con otros actores como el Estado y los movimientos sociales, sobre todo en el caso de la UNQ y movimientos sociales y la cooperación internacional, en el caso de la UNMSM.

Entonces, frente a la pregunta: de si existen experiencias que muestren evidencias de vinculaciones diferentes entre universidades y economías sociales y solidarias en Latinoamérica, la respuesta es que vienen emergiendo de manera germinal esfuerzos necesarios de consolidar que no comparten, necesariamente, el canon epistemológico de carácter eurocéntrico y despliegan más bien iniciativas orientadas a ir más allá del mismo, otorgando protagonismo a los saberes producidos desde las vidas de los productores así como de sus organizaciones y movimientos sociales. Estos esfuerzos exhiben apuestas éticas, pasión romántica y la voluntad política necesaria para desenvolver estas vinculaciones.

Una anotación final y necesaria es que si bien, como señalaba Coraggio, es preciso que haya sujetos colectivos comunitarios y sociales que planteen sus necesidades de formación, conocimiento y servicios, en una relación dialógica institucionalizada, es necesario que sea abierta a la innovación y el aprendizaje para permear al conjunto de la universidad. También es necesario e indispensable que existan actores universitarios que busquen recoger estas necesidades y posean la sensibilidad suficiente para tejer estas relaciones dialógicas institucionalizadas, igualmente abiertas a la innovación y el aprendizaje y con un conocimiento de la dinámica interna de la universidad que facilite el tejido de estas relaciones y lo ponga al servicio de las necesidades planteadas de los sujetos colectivos comunitarios y sociales.

Estos actores universitarios igual que los sujetos colectivos comunitarios desenvuelven prácticas y producen discursos que también potencialmente pueden permear al conjunto de la universidad; no son, por lo tanto, prescindibles de una lectura de conjunto de los procesos analizados; y mucho menos pueden estar ausentes de procesos de intervención como los evidenciados a través de las experiencias mostradas en los casos de la UNQ y la UNMSM. Por 
lo tanto, además de los sujetos colectivos comunitarios es necesario reconocer a los actores universitarios interesados en desenvolver las experiencias de vinculación entre universidades y economías sociales y solidarias. Es preciso incluso estrategias de fomento y hasta de fortalecimiento de estos actores, en la perspectiva de que pueden resultar claves en las dinámicas antes evidenciadas.

Un desafío a futuro es que las experiencias desenvueltas a partir de las relaciones entre universidades y economía sociales y solidarias, que reúnen a actores universitarios con sujetos colectivos comunitarios y sociales interesados en desenvolver estas experiencias, no constituyan esfuerzos aislados y circunscritos a las fronteras nacionales o ámbitos localizados, sino comiencen a enhebrarse a través de redes de escala regional y global, donde sus anclajes territoriales y las experiencias país desenvueltas puedan compartirse y ponerse en diálogo.

Resulta indudable que las relaciones entre universidad y economía social y solidaria pueden ser evidenciadas a través de muchas otras historias, como las aquí contadas; pero las dos experiencias analizadas consideramos son ilustrativas de las vinculaciones desenvueltas actualmente, y que desde la presente narración han intentado ser retratadas como la expresión de esfuerzos desplegados desde la vida cotidiana de los universitarios y los productores de las organizaciones y movimientos sociales de la economía social y solidaria de la Latinoamérica de hoy.

\section{NOTAS}

1 «La economía social y solidaria es — siguiendo a Coraggio (2011) — una forma de producir sociedad y no solo utilidades económicas, porque genera valores de uso para satisfacer necesidades de los mismos productores o de sus comunidades - generalmente de base territorial, étnica, social o cultural - y no está orientada por la ganancia y la acumulación de capital sin límites. Vuelve a unir producción y reproducción, al producir para satisfacer de manera más directa y mejor las necesidades acordadas como legítimas por la misma sociedad [...] Su fundamento es, sin duda, el trabajo y el conocimiento encarnado en los trabajadores y sus sistemas de organización» (pp. 45-46). Nos parece pertinente consignar la indicación de Quijano (2011) respecto a la noción economía solidaria: «La cooperativa es su institución central, alternativa al capital [...] Su diferenciación con las empresas capitalistas no se refiere, por lo mismo, ni a su división del trabajo ni a su relación con el mercado ni al lugar del salario o de la administración jerarquizada. Según sus actuales defensores, las diferencias residen, ante todo, en que sus agentes se identifican, explícitamente, como un sistema de autogestión de los trabajadores, de su fuerza de trabajo, de los instrumentos de producción, de los recursos u objetos de producción y de los productos. Es decir, se ubican ideológica y políticamente, de modo explícito, en contraposición al capitalismo». (p. 379).

2 Es pertinente mencionar que antes del desenvolvimiento de lecturas críticas como las de Coraggio, existió una larga, extendida y diversa suma de esfuerzos centrados en la relación entre universidades, trabajadores y cooperativas en muchos lugares de Latinoamérica, lo que Pastore (2010) llama la economía social tradicional o histórica, tanto desde la perspectiva de la implementación de programas de formación, experiencias de extensión universitaria e investigaciones dedicadas a las cooperativas, la participación de los trabajadores y la autogestión obrera. Señalamos los casos emblemáticos de las universidades populares, en las primeras décadas del siglo $\mathrm{xx}$, implementadas en el marco del surgimiento del movimiento estudiantil reformista en países como México, Puerto Rico, Argentina, Guatemala, Perú; las varias experiencias de extensión universitaria animadas desde fines de los años cincuenta, que cuestionan el sesgo asistencial o paternalista, y donde confluyen iniciativas institucionales universitarias junto a una creciente radicalización política del movimiento estudiantil y demandas del movimiento obrero. Pastore, Baliño y Rosas (2012) brindan una aproximación al respecto. 
3 Este aspecto en particular es trabajado con agudeza por Germaná (2013) cuando señala que para los dominados y explotados la descolonialidad de la universidad es el cuestionamiento radical a la colonialidad de la universidad. Cuestionamiento que pasa por tres principios: primero, la superación de la hegemonía de las estructuras eurocéntricas del saber; segundo, la superación de las estructuras institucionales disciplinarias; y, tercero, por el diálogo entre el saber académico y los saberes de los grupos sociales subalternizados por la colonialidad del poder (p. 13). Castro-Gómez (2007) aborda también este asunto y propone que «descolonizar la universidad» significa dos cosas: el favorecimiento de la transdisciplinariedad y la transculturalidad (pp. 89-90).

$4 \mathrm{Al}$ respecto coincidimos con una afirmación de Leher (2010), basado en el caso brasileño, él plantea: «Las reconfiguraciones en curso en las instituciones universitarias son de tal magnitud que resulta un error insistir en que el problema está relacionado tan solo con la presencia o la ausencia del Estado en la educación superior». (p. 20).

5 Nos parece necesario ubicar estas tensiones como parte del conflicto entre tendencias de reoriginalización cultural y represión/reabsorción que Quijano (1997) considera han impregnado nuestra más profunda experiencia histórica, porque no solamente subyace a nuestros problemas de identidad, sino que atraviesa todas nuestra historia, desde el comienzo mismo de la constitución de América, como una tensión continua de la subjetividad, donde el carácter del imaginario y de los modos de conocer y de producir conocimiento son una cuestión abierta y conflictiva.

6 Anotamos que existe amplia literatura dedicada a estas experiencias, los primeros trabajos donde se indaga en ellas fueron escritos a comienzos del 2000, como los textos de Singer (2011), publicado inicialmente el 2002, y Cruz (2004).

7 Datos más recientes muestran que en el 2013 la exportación de café peruano, a través del comercio justo, alcanzó los USD 118 millones; y la exportación total de productos USD 700 millones. La mitad del valor total de las exportaciones de café sigue proviniendo del comercio justo. Véase http://larepublica. pe/26-03-2014/peru-es-lider-mundial-en-exportaciones-de-cafe-de-comercio-justo.

8 La información demográfica oficial disponible indica que posee una población de 518.788 habitantes de los cuales 101.028 viven en las zonas de villas, asentamientos o viviendas en zonas precarias; es decir, el 19,5\% se encuentra en situación de vulnerabilidad social. 83.752 personas, es decir, $16 \%$ viven por lo menos con una necesidad básica insatisfechas (NBI) que lo ubica en una situación de pobreza estructural (Municipio de Quilmes, 2010, p. 7).

9 Es pertinente señalar que esta universidad adopta como base de su organización académica la estructura departamental con el objeto de proporcionar orientación sistemática a las actividades docentes y de investigación, mediante el agrupamiento de disciplinas afines y la comunicación entre docentes y estudiantes de distintas carreras. Tiene 3 departamentos y 35 carreras, 3 doctorados y 12 maestrías, además de diplomas y especializaciones. Su población está estimada en 12.500 estudiantes.

10 La UNMSM está organizada por facultades y a diferencia de la UNQ no ha incorporado en su discurso académico institucional la superación de la compartamentalización y especialización por campos disciplinarios.

11 Quijano precisa al respecto, años después, que los esfuerzos de los trabajadores «marginalizados» por resolver sus problemas de sobrevivencia y, en consecuencia, por motivaciones de eficacia práctica, se han ido orientando en muchos casos, cuyo número parece ir en aumento, a fortalecer y a expandir las relaciones económicas de reciprocidad o intercambio de fuerza de trabajo y o de trabajo sin pasar por el mercado; a organizarse colectivamente de modo «comunal», en lugar de empresarial, para gestionar adecuadamente sus recursos, sus productos, sus relaciones en el mercado y con los otros sectores de la economía y de la sociedad. Lo que caracteriza la economía popular es justamente que las relaciones de trabajo y de distribución de recursos y del producto se organizan, en lo fundamental, en torno a la reciprocidad y la vida social, las prácticas sociales cotidianas y la comunidad. Eso no significa, por su supuesto, que no esté articulada con el mercado de múltiples maneras y medidas (Quijano, 2011, p. 380). 12 El Comité Asesor es una instancia de deliberación y toma de decisiones, reúne representantes de docentes y estudiantes de la escuela elegidos para este fin. Las escuelas académico profesionales son instan- 
cias que incluyen a los departamentos y son dirigidas a nivel académico y administrativo por directores elegidos por los consejos de facultad. El artículo 105 del Estatuto de la UNMSM establece entre otras atribuciones del Comité Asesor: planificar y evaluar el funcionamiento de la Escuela Académico Profesional.

\section{REFERENCIAS}

Acosta, M. y Levin, A. (2013). La formación universitaria en cooperativismo y economía social en Argentina. Buenos Aires, Argentina: Centro de Estudios de Sociología del Trabajo, UBA.

Altschuler, B., Schmalko, N. y Zeballos, T. (2011, noviembre). Economía social y extensión universitaria. Formación para la autogestión en articulación con políticas públicas de inclusión social. Ponencia presentada al xi Congreso iberoamericano de extensión universitaria. Santa Fe, Argentina.

Asamblea Nacional de Rectores (2011). Datos estadísticos universitarios. Lima, Perú: Dirección de Estadística de la Asamblea Nacional de Rectores.

Baliño, G. y Rosas, G. (2012, noviembre). La extensión universitaria vinculada a organizaciones cooperativas y de economía social y solidaria. Algunas reflexiones para el debate. Informe presentado en el seminario interno del Programa Crees-icotea. Buenos Aires, Argentina.

Cárdenas, G. (1983). El sector de economía social en el Perú. Cooperativas y empresas autogestionarias. Lima, Perú: CEDEP.

Castro-Gómez, S. (2007). Decolonizar la universidad. La hybris del punto cero y el diálogo de saberes. En S. Castro-Gómez y R. Grosfoguel (Eds.), El giro decolonial. Reflexiones para una diversidad epistémica más allá del capitalismo global. Bogotá, Colombia: Iesco-Pensar-Siglo del Hombre Editores.

Coraggio, J. (2001, septiembre). Construir universidad en la adversidad. Desafíos de la educación superior en América Latina. Ponencia en la ir Reunión de Ministros de Educación de las Américas en el Ámbito del Cidi, Punta del Este, Uruguay.

Coraggio, J. (2002, julio). Universidad y desarrollo local. Ponencia presentada en el Seminario Internacional La educación superior y las nuevas tendencias. Quito, Ecuador.

Coraggio, J. (2011). La Economía Social como vía para otro desarrollo social. En A. Acosta y E. Martínez (Eds.), José Luis Coraggio. Economía Social y Solidaria. El trabajo antes que el capital. Quito, Ecuador: Abya Yala.

Coraggio, J. (2014, diciembre). La Economía Social y Solidaria: El papel de las universidades. Ponencia presentada en el seminario Universidad pública y economías solidarias, organizado por el Seminario de Economía Social, Solidaria y Popular. Lima, Perú.

Cruz, A. (diciembre, 2004). É caminhando que se faz o camino: diferentes metodologias das incubadoras tecnológicas de cooperativas populares no Brasil. Cayapa. Revista Venezolana de Economía Social, 4(8), 38-57.

Fidel, C., Di Tomaso, R. y Farías, C. (2008). Territorio, condiciones de vida y exclusión: El Partido de Quilmes (Provincia de Buenos Aires, Argentina). Buenos Aires, Argentina: ClAcso.

García Müller, A. (2007, mayo-junio). Universidad y educación cooperativa en Venezuela: experiencias. En Universidad y educación cooperativa en Venezuela, Memorias del Primer Coloquio Nacional «Universidad y Educación Cooperativa». Trujillo, Venezuela.

Germaná, C. (2013, enero). Iniciativas universitarias para la economía social y solidaria en el Perú. Ponencia presentada en el seminario Derechos económicos y políticas públicas de economía social y solidaria: retos para la universidad desde el suma qamaña. La Paz, Bolivia. 
Instituto Nacional de Estadística e Informática (2012). Censo Nacional Universitario 2010. Recuperado de http://censos.inei.gob.pe/cenaun/redatam_inei/

Izquierdo Muciño, M. (julio, 2009). Problemas de las empresas cooperativas en México que atentan contra su naturaleza especial. Boletín de la Asociación Internacional de Derecho Cooperativo, 43, 93-123.

Leher, R. (2010). Capitalismo dependiente y educación: propuestas para la problemática universitaria. En R. Leher (Comp.), Por una reforma radical de las universidades latinoamericanas. Rosario Santa Fe, Argentina: CLACso-Homo Sapiens Ediciones.

López, S. (2013). Acción universitaria para refundar San Marcos. En Por un acuerdo para refundar la Facultad de Ciencias Sociales y la Universidad de San Marcos. Lima, Perú: Universidad Nacional Mayor de San Marcos.

Mogrovejo, R., Vanhuynegem, P. y Vásquez, M. (2012). El renacimiento del modelo cooperativo peruano. En R. Mogrovejo, P. Vanhuynegem y M. Vásquez (Eds.), El cooperativismo en América Latina. Una diversidad de contribuciones al desarrollo sostenible. La Paz, Bolivia: OIт.

Municipio de Quilmes (2010). Censo social Quilmes. Resultados preliminares. Quilmes, Argentina: Secretaría de Desarrollo Social del Municipio de Quilmes.

Pastore, R. (octubre, 2010). Un panorama del resurgimiento de la economía social y solidaria en Argentina. Revista de Ciencias Sociales, 2(18), 47-74.

Pérez Alva, E. (2014, noviembre). Bordando la vida desde otras economías. Ponencia presentada en el Encuentro de vinculación socioeconómica, desarrollo socio-técnico e incubadoras universitarias. Buenos Aires, Argentina.

Quijano, A. (1977). Imperialismo y «marginalidad» en América Latina. Lima, Perú: Mosca Azul Editores.

Quijano, A. (1997). Colonialidad del poder, cultura y conocimiento en América Latina. Anuario Mariateguiano, $\operatorname{Ix}(9), 113-122$.

Quijano, A. (2011). ¿Sistemas alternativos de producción? En B. Santos (Coord.), Producir para vivir. Los caminos de la producción no capitalista. México DF, México: Fondo de Cultura Económica.

Remy Simatovic, M. I. (2007). Cafetaleros empresarios. Dinamismo asociativo para el desarrollo en el Perú. Lima, Perú: Instituto de Estudios Peruanos, Oxfam Internacional.

Rénique, J. (marzo, 1987). De la fe en el progreso al mito andino: Los intelectuales cusqueños. Márgenes, $1,9-33$.

Rofman, A. (2005). Introducción. En A. Rofman (Comp.), Universidad y desarrollo local. Aprendizajes y desafíos, Buenos Aires, Argentina: Prometeo Libros.

Sena, S., Baliño, G. y Martínez, M. (2012). En un diálogo de saberes: organizaciones sociales, universidad, economía social y solidaria. En II Jornada de Investigadores sobre Aprendizaje-Servicio. Buenos Aires, Argentina: Centro Latinoamericano de Aprendizaje y Servicio Solidario, Red Iberoamericana de aprendizaje-servicio.

Singer, P. (2011). La reciente resurrección de la economía solidaria en Brasil. En B. Santos (Coord.), Producir para vivir. Los caminos de la producción no capitalista. México DF, México: Fondo de Cultura Económica.

Tillmann, R., Della Vechia R. y Nunes, T. (abril, 2011). La Red de Incubadoras Tecnológicas de Cooperativas Populares en Brasil: pasado, presente y desafíos para el futuro. Revista del Centro de Estudios de Sociología del Trabajo, 3, 1-27. 